\title{
MiR-139-5p inhibits migration and invasion of colorectal cancer by downregulating AMFR and NOTCH1
}

\author{
Mingxu Song ${ }^{1}$, Yuan Yin ${ }^{1}$, Jiwei Zhang ${ }^{1}$, Binbin Zhang ${ }^{1}$, Zehua Bian ${ }^{1}$, Chao Quan ${ }^{1}$, Leyuan Zhou ${ }^{3}$, \\ Yaling $\mathrm{Hu}^{1}$, Qifeng Wang ${ }^{4}$, Shujuan $\mathrm{Ni}^{4}$, Bojian $\mathrm{Fei}^{2}$, Weili Wang ${ }^{2}$, Xiang $\mathrm{Du}^{4}$, Dong Hua ${ }^{1}$, Zhaohui Huang ${ }^{1 凶}$ \\ ${ }^{1}$ Wuxi Oncology Institute, the Affiliated Hospital of Jiangnan University, Wuxi 214062, China \\ 2 Department of Surgical Oncology, the Affiliated Hospital of Jiangnan University, Wuxi 214062, China \\ ${ }^{3}$ Department of Radiation Oncology, the Affiliated Hospital of Jiangnan University, Wuxi 214062, China \\ ${ }^{4}$ Department of Pathology, Fudan University Shanghai Cancer Center, Shanghai 200032, China \\ $\triangle$ Correspondence: hzhwxsy@126.com (Z. Huang) \\ Received June 14, 2014 Accepted July 14, 2014
}

\begin{abstract}
MicroRNAs (miRNAs) that exert function by posttranscriptional suppression have recently brought insight in our understanding of the role of non-protein-coding RNAs in carcinogenesis and metastasis. In this study, we described the function and molecular mechanism of miR-139-5p in colorectal cancer (CRC) and its potential clinical application in CRC. We found that miR-139-5p was significantly downregulated in $73.8 \%$ CRC samples compared with adjacent noncancerous tissues (NCTs), and decreased miR-139-5p was associated with poor prognosis. Functional analyses demonstrated that ectopic expression of miR-139-5p suppressed CRC cell migration and invasion in vitro and metastasis in vivo. Mechanistic investigations revealed that miR-139-5p suppress CRC cell invasion and metastasis by targeting AMFR and NOTCH1. Knockdown of the two genes phenocopied the inhibitory effect of miR-139-5p on CRC metastasis. Furthermore, the protein levels of the two genes were upregulated in CRC samples compared with NCTs, and inversely correlated with the miR-139-5p expression. Increased NOTCH1 protein expression was correlated with poor prognosis of CRC patients. Together, our data indicate that miR-139-5p is a potential
\end{abstract}

Mingxu Song, Yuan Yin these two authors contributed equally to this work.

Electronic supplementary material The online version of this article (doi:10.1007/s13238-014-0093-5) contains supplementary material, which is available to authorized users. tumor suppressor and prognostic factor for CRC, and targeting miR-139-5p may repress the metastasis of CRC and improve survival.

KEYWORDS miR-139-5p, AMFR, NOTCH1, colorectal cancer, metastasis, prognosis

\section{INTRODUCTION}

MicroRNAs (miRNAs) are small endogenous non-coding RNAs that regulate the expression of target genes at the post-transcriptional level through sequence-specific binding to the $3^{\prime}$ untranslated region ( $\left.3^{\prime} U T R\right)$ of their specific mRNAs, resulting in translational inhibition even destruction of the target mRNAs (Bartel 2004). MiRNAs play vital roles in tumorigenesis by acting as tumor suppressors, such as let-7, miR-143, and miR-200 (Chen et al. 2009; Li et al. 2009), or oncogenes, such as miR-21, miR-155, and miR-95 (Asangani et al. 2008; Huang et al. 2011; Hwang et al. 2014). Deregulation of miRNAs is a common event in human tumors and involves in various pathological processes, possessing with either oncogenicity or promotion of metastasis. Though recent researches of miRNAs have brought mind-blowing insight into our knowledge of human cancers, there are still largely unknown details that need to be explored further.

Colorectal cancer (CRC) is one of the most commonly diagnosed cancers in both genders worldwide. Over 1.2 million new cancer cases occur annually, resulting in greater than 600,000 deaths each year (Jemal et al. 2011). Although some advances are achieved in the diagnosis and treatment, 


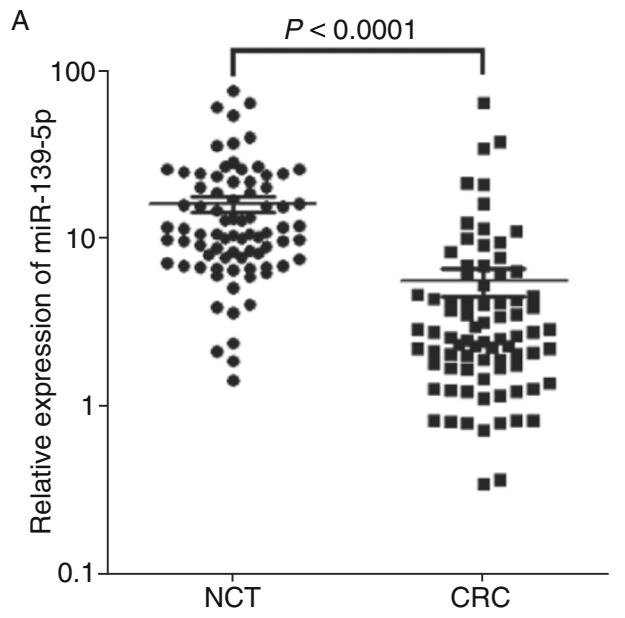

B

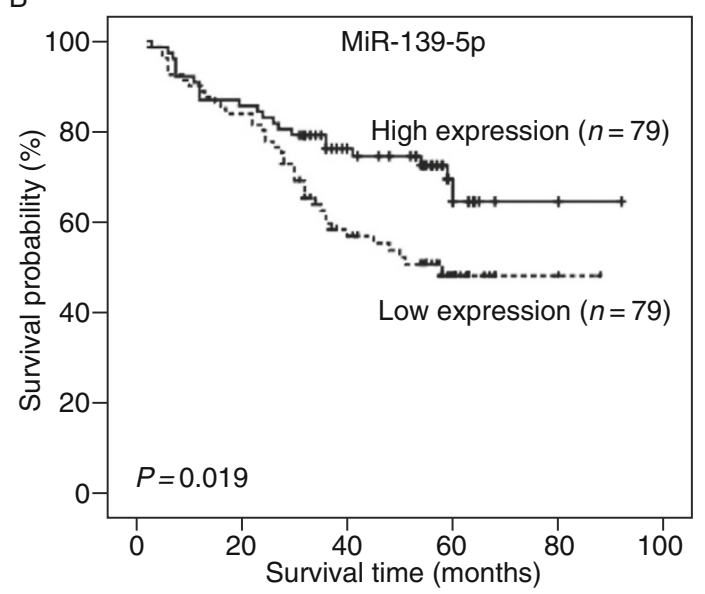

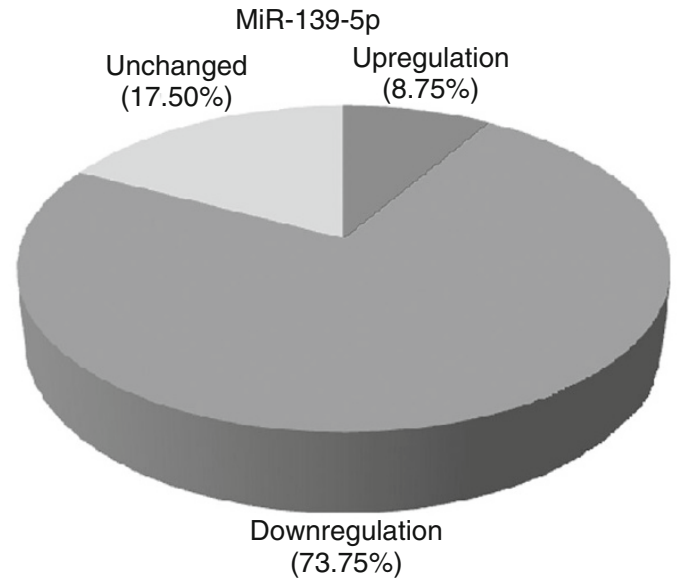

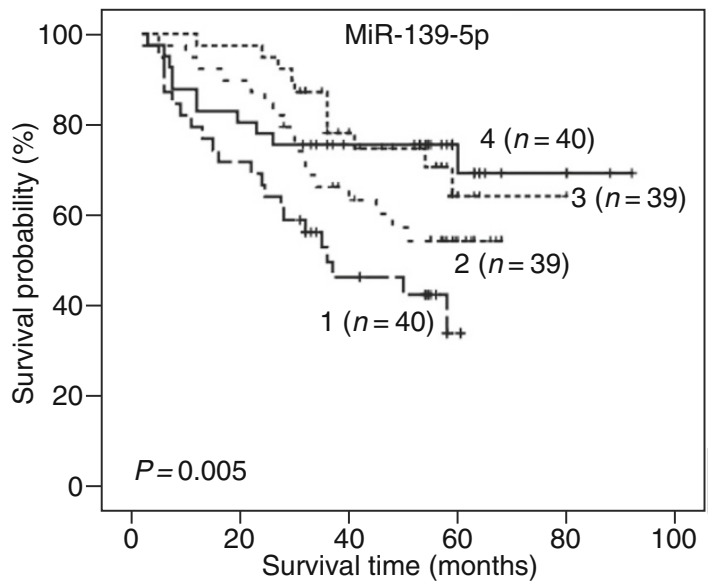

Figure 1. miR-139-5p is frequently downregulated and associated with poor overall survival in CRC. (A) MiR-139-5p expression was detected by quantitative reverse transcription polymerase chain reaction (qRT-PCR) in 80 paired CRC and adjacent noncancerous tissues (NCTs). MiR-139-5p expression was markedly downregulated in tumor tissues compared with the corresponding NCTs (U6 small nuclear RNA was used as an internal control). (B) Overall survival analysis based on the expression level of miR-139-5p. MiR-139-5p expression was examined in $158 \mathrm{CRC}$ tissues, and these cases were divided into two groups (high or low) or four groups (1-4) based on their miR-139-5p levels in tumors. MiR-139-5p expression was positively correlated with the overall survival.

the disease is very often diagnosed at an advanced stage with tumor metastasis, making it starving for the discovery of new metastasis relevant molecules that could help to improve the diagnosis and prognosis.

Many studies have shown that certain miRNAs play important roles in various processes of the metastatic cascade, such as cell adhesion, migration, invasion, angiogenesis, and epithelial-mesenchymal transition (EMT) (Hurst et al. 2009; Nicoloso et al. 2009). However, detailed mechanisms that contribute to metastasis process remain poorly understood. Our previous data revealed that miR139-5p was downregulated in CRC (Huang et al. 2011). Interestingly, decreased expression of miR-139-5p has also been found in several other tumors, including breast cancer, gastric cancer, hepatocellular carcinoma, glioblastoma, and laryngeal squamous carcinoma (Guo et al. 2009; Gu et al. 2013; Krishnan et al. 2013; Li et al. 2013; Liu et al. 2013). Recent data suggested that miR-139 may inhibit CRC growth and/or metastasis by targeting different genes (Guo et al. 2012; Shen et al. 2012; Zhang et al. 2014). However, their results were contradictory. The accurate role and clinical value of miR-139-5p in CRC remain to be unclear. In this study, we confirmed the downregulation of miR-139-5p in an expanded CRC cohort, which correlated with poor survival of CRC patients, and revealed that miR-139-5p could inhibit CRC invasion and metastasis through directly targeting AMFR and $\mathrm{NOTCH} 1$ in $\mathrm{CRC}$. 

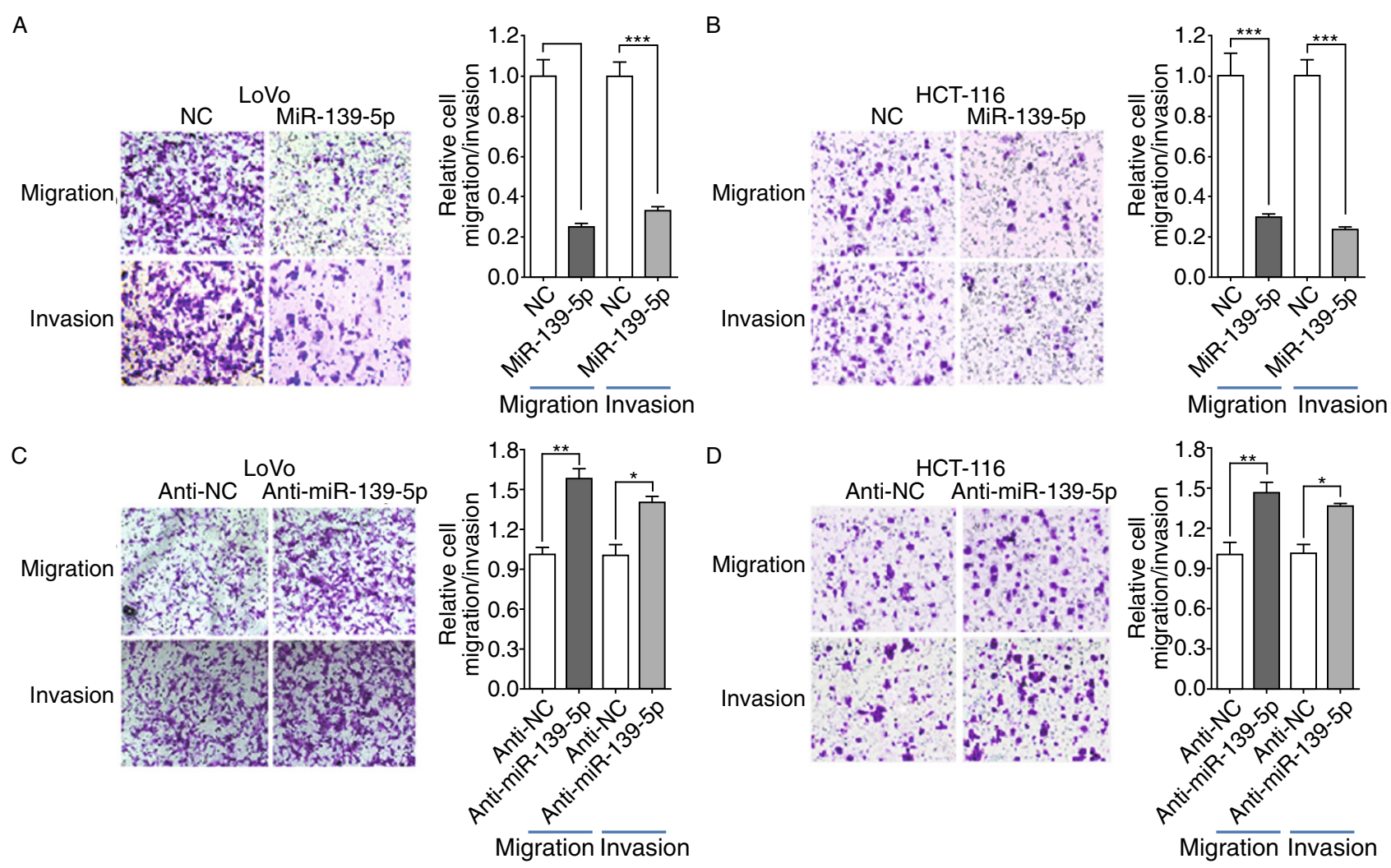

E
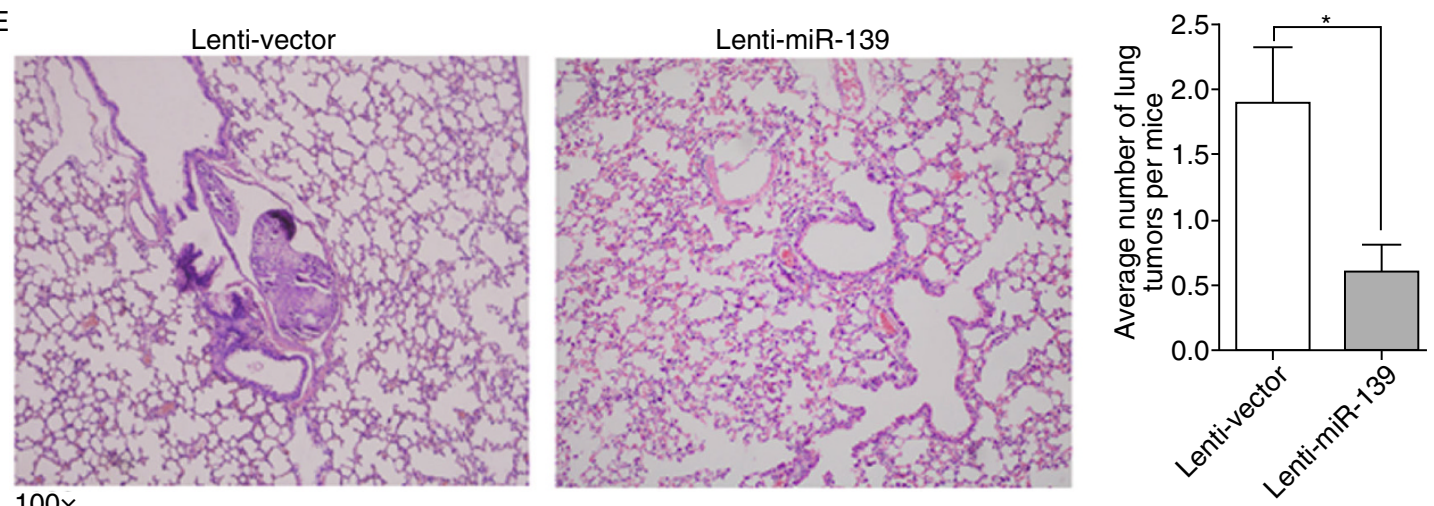

Figure 2. MiR-139-5p inhibits CRC cell migration and invasion in vitro and in vivo. (A and B) The overexpression of miR-139-5p inhibits the migration and invasion of LoVo and HCT-116 cells. (C and D) The silencing of miR-139-5p enhanced the migration and invasion of LoVo and HCT-116 cells. (E) The effect of miR-139 on tumor metastasis in a lung metastasis model of nude mouse. LoVo cells stably expressing miR-139 or the control $\left(2 \times 10^{6}\right)$ were injected into the caudal vein of each nude mouse. The ectopic expression of miR-139 significantly reduced the number of lung metastases $\left({ }^{\star} P<0.05\right)$.

\section{RESULTS}

Decreased miR-139-5p expression is associated with poor overall survival in CRC

Our data of miRNA expression profile have demonstrated that miR-139-5p is downregulated in CRCs compared to NCTs (Huang et al. 2011). To further verify the result, the expression level of miR-139-5p was examined using qRT-
PCR in an expanded cohort of 158 CRCs, including 80 tumors with paired NCTs. In accordance with the microarray data, the results showed that miR-139-5p was downregulated more than 2 -fold in $73.8 \%$ of CRCs compared with NCTs $(P<0.0001$, Fig. $1 \mathrm{~A})$. Survival analysis showed that high miR-139-5p expression was associated with prolonged overall survival of CRC patients (high: the $25 \%$ highest, low: the $25 \%$ lowest) $(P<0.05$, Fig. 1B). After adjustment for age, 
gender, tumor size, pathologic stage, and grading, a Cox multivariate analysis indicated that miR-139-5p expression is an independent prognostic factor for CRC (adjusted $\mathrm{HR}=$ $2.681,95 \% \mathrm{Cl}=1.532-4.691, P=0.001$, Table S1). No significant relationship was found between miR-139-5p expression in CRC and tumor size, location, stage, and grading $(P>0.05)$.

\section{Overexpression of miR-139-5p inhibits CRC cell migration and invasion in vitro and metastasis in vivo}

Now that miR-139-5p was downregulated in CRC and associated with poor prognosis, we conclude that miR-139$5 p$ may have an inhibitory role on CRC cell proliferation and/ or invasion. To determine whether miR-139-5p could regulate the metastasis ability of $\mathrm{CRC}$, we first examined the effect of miR-139-5p on CRC cell mobility. The results showed that miR-139-5p overexpression can suppress the cell migration and invasion (Fig. $2 \mathrm{~A}$ and $2 \mathrm{~B}$ ), whereas silencing of miR-139-5p promotes cell migration and invasion (Fig. 2C and 2D). To identify the impact of miR-139-5p on in vivo metastasis of CRC cells, LoVo cells stably expressing miR-139-5p or the vector control were injected into the caudal vein of athymic BALB/c nude mice. Ectopic expression of miR-139-5p significantly reduced the number of lung metastasis sites (Fig. 2E and Table S2), further validating that the invasive behaviour of CRC could be suppressed by miR-139-5p. In contrast, no significant effect of miR-139-5p on cell proliferation was observed based on the in vitro and in vivo analyses (Fig. S1). Because pri-miR-139 could produce both miR-139-3p and miR-139-5p, we checked the expression and function of miR-139-3p in CRC cells. The results showed that the expression of miR-139-3p in CRC cells was much lower than that of miR-139-5p (Fig. S2A and S2B), and overexpression of miR-139-3p showed no significant effect on CRC cell growth and migration (data not shown). Collectively, these data indicate that miR-139-5p was able to suppress CRC migration and invasion.

\section{AMFR and NOTCH1 are the direct target genes of miR- 139-5p in CRC}

To investigate the mechanism by which miR-139-5p exerts anti-metastasis function in $\mathrm{CRC}$, we searched for the potential targets of miR-139-5p using bioinformatics algorithms (TargetScan and miRanda) and a microarray assay. Genomic-wide expression profiling was performed in NCand miR-139-5p-transfected LoVo cells using a microarray. A total of 1443 downregulated transcripts (>2-fold change) were identified in miR-139-5p-transfected cells compared with the control (Table S3). Among the top 500 targets of miR-139-5p predicted by the two algorithms, 31 genes were downregulated in the miR-139-5p-transfected cells. Then, 5 potential target genes with tumor-promoting function (AMFR, NOTCH1, HNRNPF, TOP1, and LAPTM4B) were selected from the 31 genes and their $3^{\prime} U T R s$ containing the
Figure 3. AMFR and NOTCH1 are the direct target genes of miR-139-5p. (A) Initial screening of miR-139-5p target genes using a microarray assay, bioinformatics predictions and the luciferase reporter assay. Five downregulated genes (AMFR, NOTCH1, HNRNPF, TOP1, and LAPTM4B) were selected from the 31 genes in the initial screening based on the functional analysis of these genes, and their $3^{\prime} U T R$ s were assessed using the luciferase reporter assay. ( $B$ and $C$ ) Analyses of the luciferase activity of the luciferase reporter plasmids containing either wild-type (WT) or mutant-type (MT) 3'UTRs of AMFR and NOTCH1 in HEK-293T and HCT-116 cells. A mutation was generated in the site complementary to the miR-139-5p seed region of the 3 'UTR of AMFR or NOTCH1, as indicated. (D) The protein levels of AMFR and NOTCH1 were determined by Western blot assays using LoVo and HCT-116 cells transfected with miR-139-5p mimic, miR-139-5p inhibitor (anti-miR139-5p) or their corresponding negative control (NC or anti-NC). Beta-actin protein was used as an internal control.

complementary binding sites of miR-139-5p were cloned into a luciferase reporter vector to evaluate the influence of miR$139-5 p$ on the expression of a reporter gene using a luciferase assay (Fig. 3A). The expression of the reporter gene in the recombinant plasmids containing AMFR and NOTCH1 3'UTRs were significantly repressed by miR-139-5p. To further confirm that AMFR and NOTCH1 are direct targets of miR-139-5p in CRC, we mutated the predicted binding sites of miR-139-5p in the 3'UTRs of AMFR and NOTCH1 that are conserved among mammals, and found that the mutant 3'UTRs were completely refractory to miR-139-5p-mediated luciferase reporter repression in HEK-293T and HCT-116 cells (Figs. 3B, 3C and S3). In line with these results, the endogenous AMFR and NOTCH1 protein levels were also decreased in miR-139-5p-overexpressing CRC cells and could be restored in miR-139-5p-depleted cells (Fig. 3D).

The protein expression of AMFR and NOTCH1 is upregulated in CRC and negatively correlated with miR139-5p expression

To further evaluate the relationship between miR-139-5p and AMFR/NOTCH1, we detected their expression in paired CRC and NCT tissues using qRT-PCR and immunohistochemistry, respectively. Brown cytoplasmic AMFR and NOTCH1 staining was strong in CRC tissues but nearly absent in the normal epithelia (Fig. 4A, 4B and S4). Of the 134 cases, 106 tumors showed increased AMFR expression and 115 tumors showed increased NOTCH1 expression compared with the paired NCTs. The protein expression of AMFR and NOTCH in tumor tissues inversely correlated with the miR-139-5p levels (AMFR: Spearman $r=-0.352, P=0.0001$; NOTCH1: Spearman $r=-0.184, P=0.033$, Fig. 4C). Interestingly, a positive correlation was observed between the protein expression of AMFR and NOTCH1 in CRC (Spearman $r=0.986, P<$ 
A
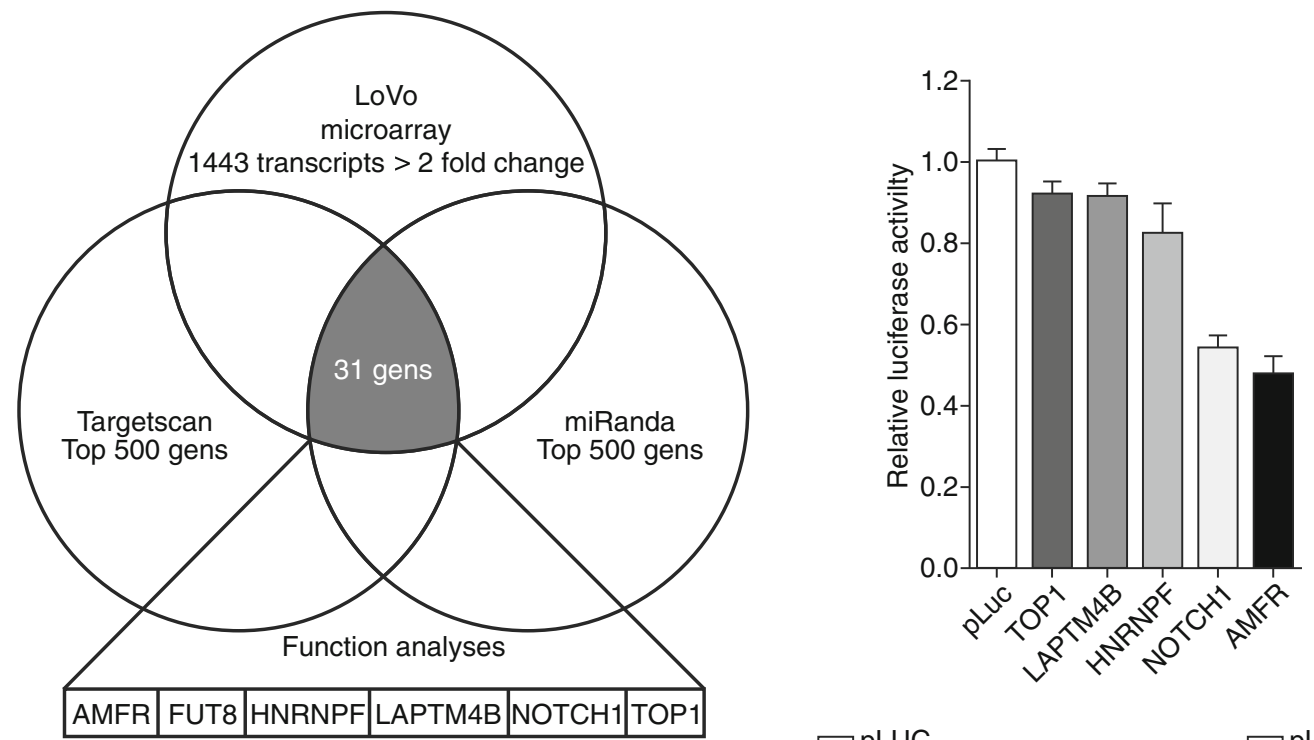

B
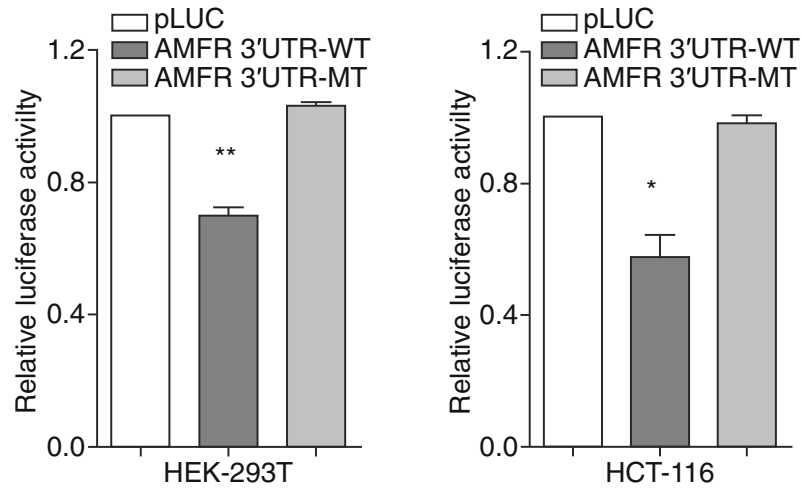

C

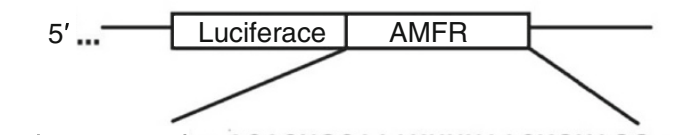

3' UTR-WT 5'.....ACACUGCAAAUUUUAACUGUAGG... MiR-139-5p 3'......GACCUCUGUGCACG-U'UI I I II I 3' UTR-MT 5' '..... ACACUGCAAAUUUUAAGACAUGG... MiR-139-5p 3'......CACCUUACCGAUUA-UGACAUCU
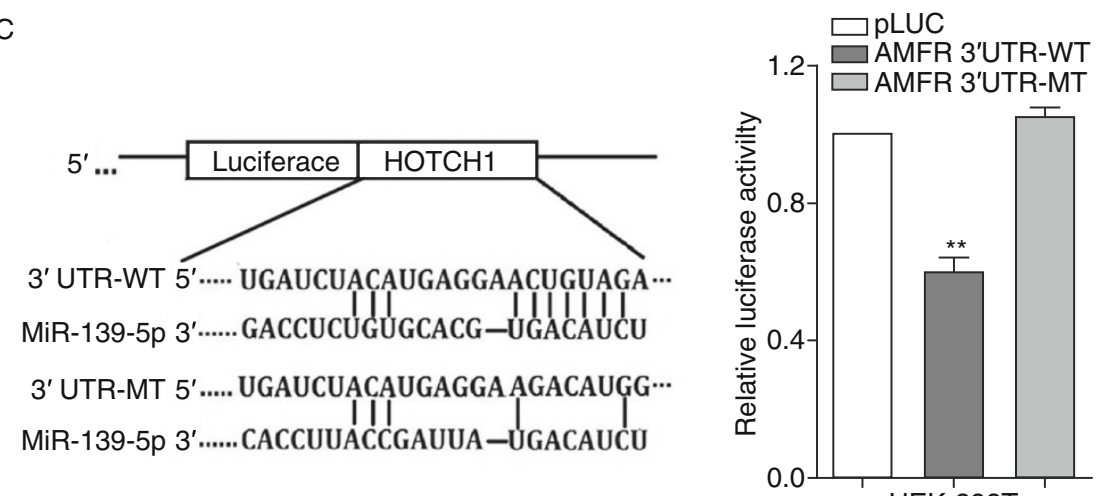

D

HEK-293T
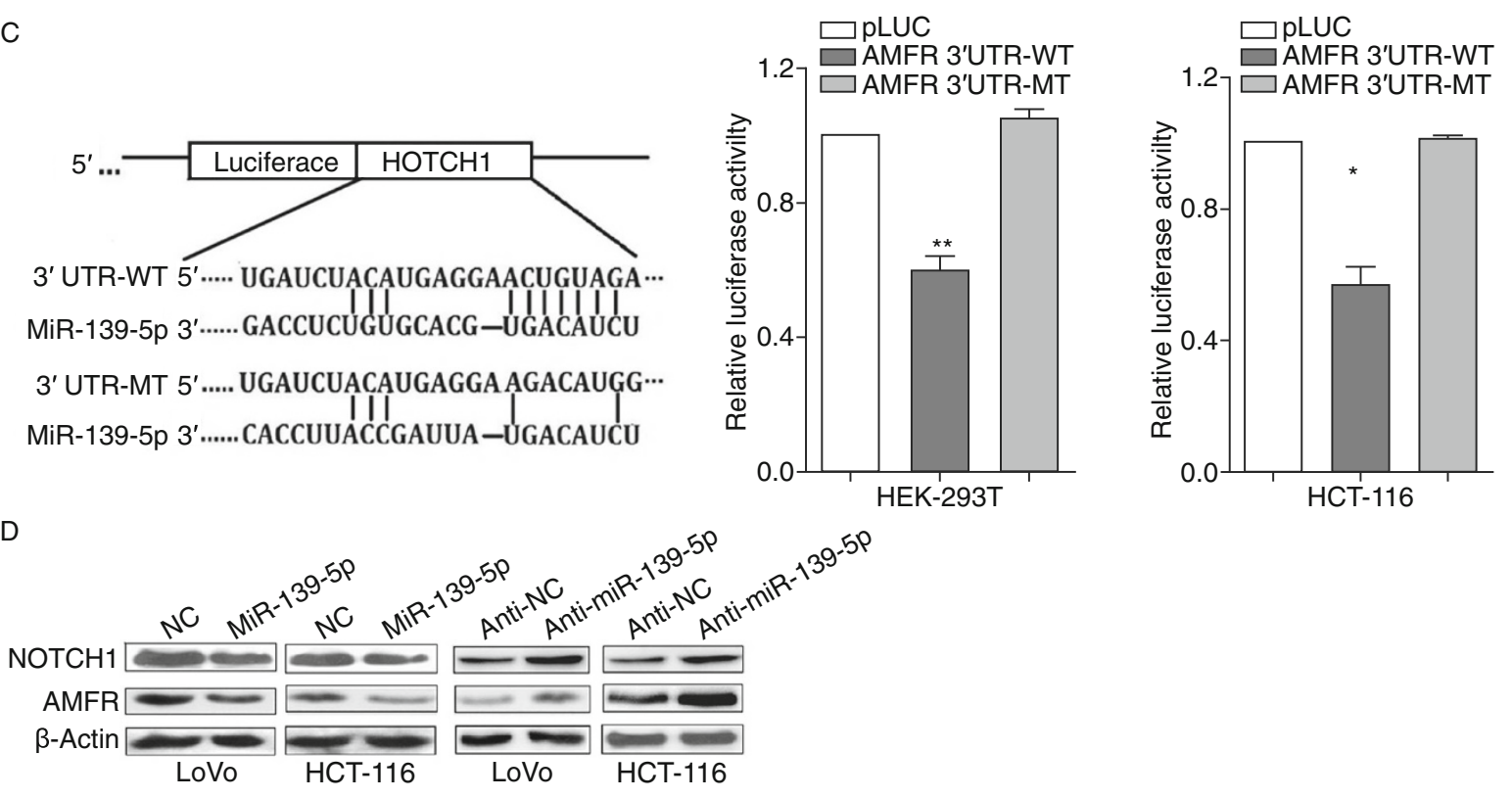
0.0001), suggesting that they may be regulated by same mechanisms. Taken together, these data suggest that miR139-5p inactivation may result in the increased expression of AMFR and NOTCH1 in human CRC.

Survival analyses revealed that increased $\mathrm{NOTCH} 1$ protein levels were associated with shorter survival time $(P<$ 0.05 , Figs. 4D and S5). After adjusting for age, gender, tumor size, tumor grading, and tumor stage, multivariate analyses showed that NOTCH1 expression was an independent risk factor for survival. Tumors with higher NOTCH1 expression (score 3) presented a higher risk of death $(\mathrm{HR}=1.984,95 \%$ $\mathrm{Cl}=1.238-3.180, P=0.004)$. No significant association was found between AMFR expression and survival.

\section{MiR-139-5p inhibits tumor invasion via directly targeting AMFR and NOTCH1 in CRC}

To examine the functional effect of AMFR and NOTCH1 in miR-139-5p-induced metastasis inhibition in CRC cells, we inhibited AMFR and NOTCH1 expression with siRNA (Fig. S6) and revealed that AMFR- and NOTCH1-depleted LoVo cells showed decreased invasion, which phenocopied the invasion-inhibiting effect of miR-139-5p. In contrast, the ectopic overexpression of AMFR and NOTCH1 using plasmids of AMFR and NOTCH1 (ICN) ORF promoted cell invasion, which could not be repressed by the overexpression of miR-139-5p (Fig. 5A and 5B). Taken together, these results proved that miR-139-5p inhibits $C R C$ invasion through AMFR and NOTCH1.

\section{DISCUSSION}

Nowadays more than 2000 miRNAs have been identified in human, which participate in keeping the balance of gene regulating networks and regulate more than a third of human genes at the post-transcription level (Esquela-Kerscher and Slack 2006). The disruption of microRNAs may be crucial in numerous cellular processes, particularly in oncogenesis and metastasis (Manikandan et al. 2008; Bouyssou et al. 2014). MiR-139-5p, one of the most significant downregulated miRNA in CRC in our previous data (Huang et al. 2011), have been identified as relating to the development of several tumor types (Bao et al. 2011; Wong et al. 2011; Krishnan et al. 2013; Liu et al. 2013), including CRC (Guo et al. 2012; Shen et al. 2012; Zhang et al. 2014). Here, we identified miR-139-5p as a metastasis inhibitor through directly targeting AMFR and NOTCH1 in CRC.

MiR-139 shows extensive function in human tumorigenesis and development. Wong first reported that miR-139 suppresses metastasis and progression of hepatocellular carcinoma by downregulating Rho-kinase 2 (Wong et al. 2011). Bao et al. revealed that HER2 and CD44 can induce histone deacetylation in the promoter region of the miR-139 gene, resulting decreased miR-139 expression and increased expression of its target CXCR4 in gastric cancer (Bao et al. 2011). Subsequent reports showed that miR-139-5p suppress the invasive capability of breast cancer (Krishnan et al. 2013) and esophageal carcinoma cells (Liu et al. 2013). These reports indicate the common key role of miR-139-5p in cancer metastasis. In this study, we confirmed the downregulation of miR-139-5p in CRC in an expanded cohort of CRC (Fig. 1A). Further functional analyses demonstrated that the overexpression of miR-139-5p can significantly suppress cell migration and invasion in vitro and metastasis in vivo (Fig. 2). These results were consistent with the results of Shen et al. and Zhang et al. (Shen et al. 2012; Zhang et al. 2014). However, Guo et al. and Zhang et al. reported that miR-139-5p could inhibit CRC cell growth in vitro (Guo et al. 2012; Zhang et al. 2014), which was not observed in the study of Shen et al. (Shen et al. 2012). We also did not observe the growthinhibiting function of miR-139-5p using in vitro and in vivo assays (Fig. S1). It may be due to different methodology or cell lines used in different studies. These conclusions should be validated under the same conditions in the future work.

AMFR, located on human chromosome $16 q^{12.2}$, known as gp78, is a ubiquitin E3 ligase involved in the degradation of proteins through the endoplasmic reticulum pathway (Fang et al. 2001). AMFR has the pluripotency activated by AMF involving cells adhesion, motility, and angiogenesis (Yanagawa et al. 2004; Chiu et al. 2008). AMF stimulates AMFR in an autocrine manner and results in signalling cascades relying on PKC and activates Rho-like GTPase, RhoA, and Rac1, which enhance cell motility (Kanbe et al. 1994; Wang et al. 2010). Several groups have shown that AMFR upregulation correlates with more advanced tumor stage and poor survival in stomach, lung, and breast cancer (Taniguchi et al. 1998; Kawanishi et al. 2000; Jiang et al. 2006), and appears to be a strong independent predictor for recurrence in CRC (Nakamori et al. 1994). Although recent data suggested that AMFR may be associated with tumorigenesis and development, the detailed function and mechanisms of AMFR remain unknown (Wang et al. 2010; Kho et al. 2013). In this study, we showed, for the first time, that AMFR was regulated by miR-139-5p and could promote CRC invasion, and the expression of AMFR protein was negatively correlated with miR-139-5p in CRC tissues, suggesting that the increasing activity of AMFR was due to the inactivation of miR-139-5p in CRC (Figs. 3 and 4). The detailed mechanism by which AMFR promotes CRC cell metastasis should be investigated in future study.

NOTCH1 is another target gene of miR-139-5p identified in CRC (Zhang et al. 2014). Four NOTCH receptors (NOTCH1-4) and five ligands (Jagged-1, 2, Delta-1, 3, 4) have been described in mammals (Miele et al. 2006). Dysregulated activity of $\mathrm{NOTCH}$ signaling is frequently observed in human cancers and a lot of studies have suggested an involvement of $\mathrm{NOTCH}$ signaling in cancer angiogenesis and metastasis (Hellstrom et al. 2007; Hu et al. 2012). $\mathrm{NOTCH} 1$ is found upregulated in $\mathrm{CRC}$ and correlates with tumor proliferation, differentiation, lymphatic metastases, and survival time (Reedijk et al. 2008; Chu et al. 2010; Hristova et al. 2013). We observed that NOTCH1 was directly regulated by $\mathrm{miR}-139-5 \mathrm{p}$ at the post-transcriptional 
A
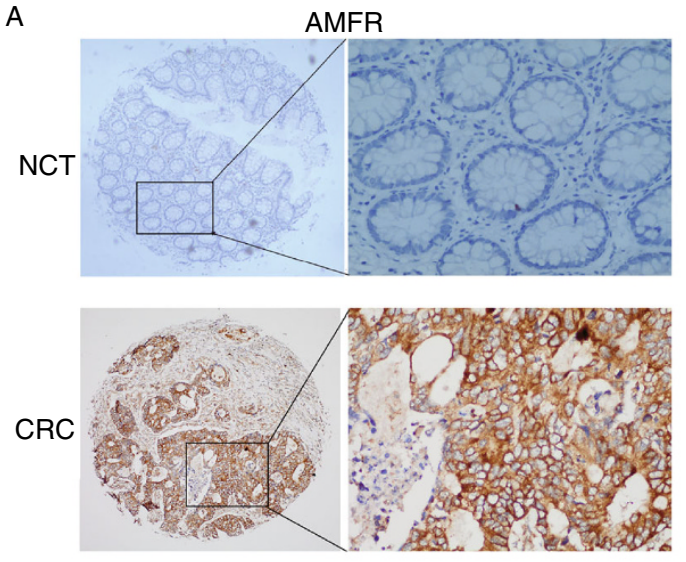

B
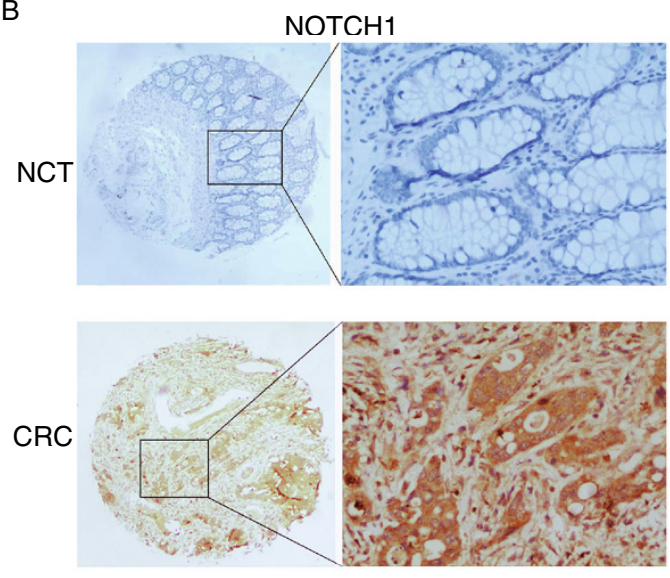

C

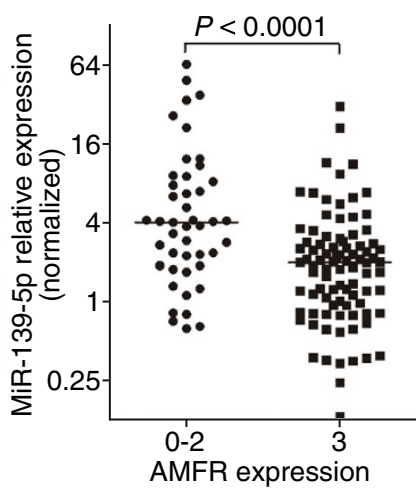

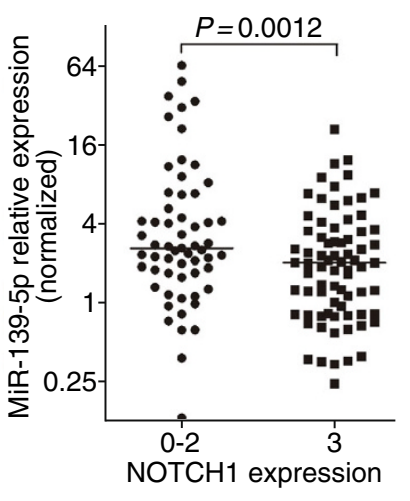

AMFR protein levels

\begin{tabular}{ccccc}
\hline & Score 0 & Score 1 & Score 2 & Score 3 \\
\hline Tumor & 0 & 9 & 38 & 87 \\
Normal & 7 & 56 & 64 & 7 \\
\hline \multicolumn{5}{c}{$\begin{array}{c}\text { Unchanged } \\
(16.42 \%)\end{array}$}
\end{tabular}

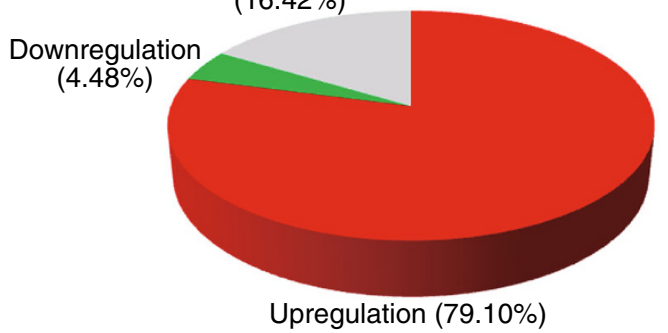

NOTCH1 protein levels

\begin{tabular}{ccccc} 
& Score 0 & Score 1 & Score 2 & Score 3 \\
\hline Tumor & 0 & 11 & 48 & 75 \\
Normal & 14 & 80 & 38 & 2 \\
\hline
\end{tabular}

Unchanged $(12.59 \%)$

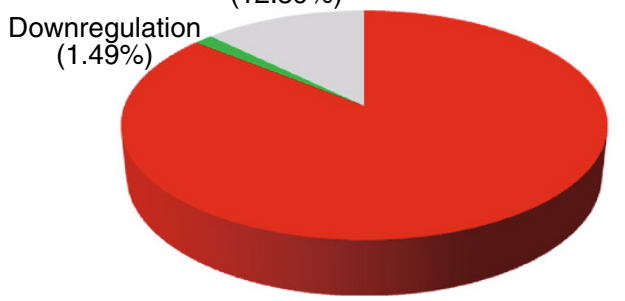

Upregulation (85.82\%)

D

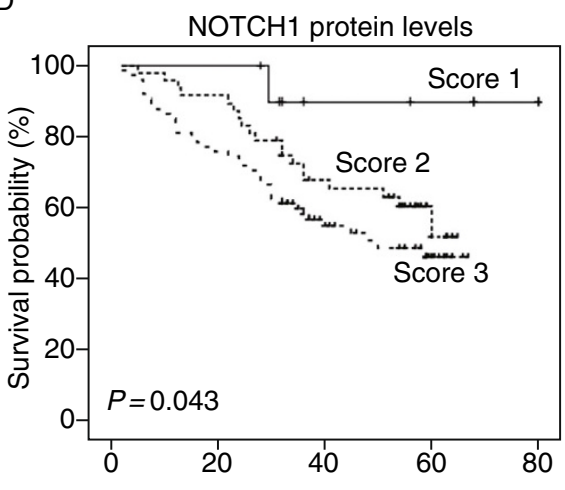

Figure 4. The protein expression of AMFR and NOTCH1 was upregulated in CRC and negatively correlated with miR-139-5p expression. (A and B) Immunohistochemical staining of AMFR and NOTCH1 in CRC tissues and corresponding noncancerous tissues (NCTs). Brown cytoplasmic AMFR/NOTCH1 staining was strong in CRC tissues but nearly absent in the normal epithelia. AMFR and NOTCH1 protein expression were frequently increased in the tumor tissues compared with the matched NCTs. (C) The expression levels of AMFR and NOTCH1 negatively correlated with the miR-139-5p levels in the CRC tissues. (D) Survival analysis based on the expression levels of $\mathrm{NOTCH} 1$ protein. The groups were ranked according to the NOTCH1 staining intensity.

level and promoted CRC invasion. And our data showed that the expression of NOTCH1 was significantly upregulated in $\mathrm{CRC}$ and negatively correlated with miR-139-5p and survival
(Figs. 3 and 4). In line with our results, the metastasisprompting function of NOTCH1 has been reported in breast cancer, prostate cancer, esophageal cancer, and melanoma 
A
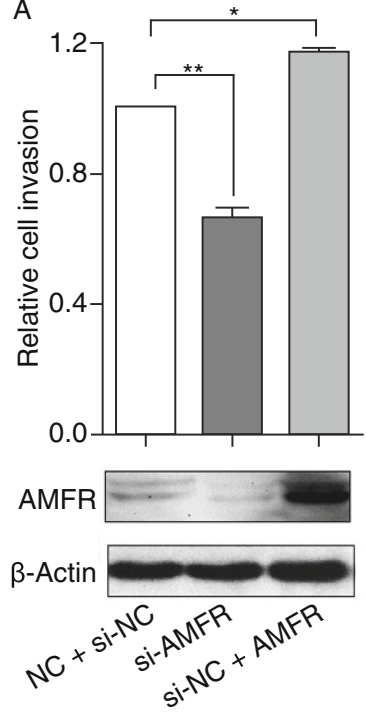
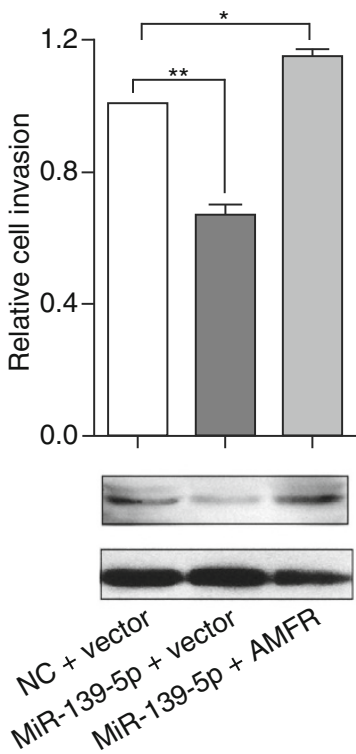
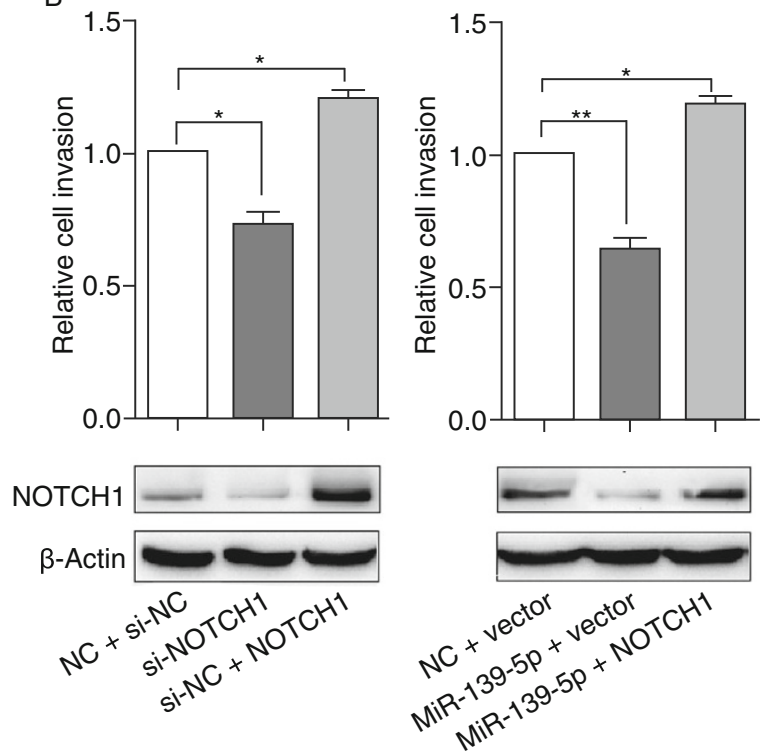

Figure 5. MiR-139-5p inhibits CRC invasion via directly targeting AMFR and NOTCH1 in CRC. (A and B) The knockdown of AMFR or NOTCH1 by siRNA significantly repressed CRC cell invasion, which phenocopied the function of miR-139-5p, whereas the overexpression of AMFR or NOTCH1 (ORF without 3'UTR) markedly promoted cell invasion and counteracted miR-139-5p-induced invasion inhibition in LoVo cells.

(Wang et al. 2010; Hu et al. 2012). In addition to miR-139-5p, other miRNAs (miR-34a, miR-144) were also reported to inhibit NOTCH1 in CRC (Sureban et al. 2011; Bu et al. 2013), indicating that there is a significant cross-talk between NOTCH and miRNAs (Mo et al. 2013).

In conclusion, we determined that miR-139-5p is downregulated in $C R C$ and appears to be a prognostic factor for CRC, and miR-139-5p inhibits CRC invasion and metastasis by targeting AMFR and NOTCH1. These data suggest that restoration of miR-139-5p may be a promising therapeutic strategy for anti-metastasis treatment of CRC.

\section{MATERIALS AND METHODS}

Human tissues and cell lines

A total of 158 human primary CRC tissues and their paired adjacent noncancerous tissues (NCTs) were collected between 2005 and 2008 at Affiliated Hospital of Jiangnan University and Fudan University Shanghai Cancer Center. The detailed clinical information for the CRC patients is included in the Supplementary Data (Table S4). All of the materials of patients were obtained with informed consent, and this project was approved by the Clinical Research Ethics Committees of the Affiliated Hospital of Jiangnan University and Fudan University Shanghai Cancer Center. HEK-293T cell line and human CRC cell lines, including HCT-8, HCT-116, LoVo, and SW480, were purchased from American Type Culture Collection (ATCC). HCT-8, HCT-116, and LoVo cells were cultured in RPMI1640, McCoy's 5a, and F12-K medium, respectively. HEK-293T and
SW480 cells were maintained in DMEM and Leibovitz's L-15 medium, respectively. All of the media (Hyclone, USA) were supplemented with $10 \%$ fetal bovine serum (Gibco, USA), plus penicillin $(100 \mathrm{U} / \mathrm{mL})$ and streptomycin $(100 \mathrm{U} / \mathrm{mL})$. The cells were incubated under the conditions recommended by ATCC.

RNA extraction and quantitative real-time RT-PCR

Total RNA was extracted using the TRIzol reagent (Invitrogen, USA) according to the manufacturer's instructions. The concentration of RNA was measured using a NanoDrop ND-1000 instrument (NanoDrop, USA). cDNA was synthesised using the PrimeScript RT reagent kit (TaKaRa, Japan). qPCR analyses were conducted to quantitate mRNA relative expression using SYBR Premix Ex Taq (TaKaRa, Japan) with beta-actin as an internal control. TaqMan microRNA assays (Applied Biosystems, USA) were used to determine miRNA expression levels (Applied Biosystems, USA) with U6 small nuclear RNA as an internal control. The results of qPCR were defined from the threshold cycle (Ct), and relative expression levels were calculated by using the $2^{-\triangle \Delta C t}$ method (Livak and Schmittgen 2001). PCR was performed using $A B I 7300 H T$ and $A B I$ Vii7 instrument (Applied Biosystems, USA). The primers used for PCR analysis were listed in the Table S5.

\section{Vector constructs}

The human pri-miR-139 sequence was amplified from normal human genomic DNA by nested PCR using PrimerSTAR Premix (TaKaRa, Japan). The sequence was then cloned into the lentivirus expression vector pWPXL (Clontech, USA) to generate pWPXL- 
miR-139. The predicted binding sites in the $3^{\prime} U T R$ s of the potential target genes of miR-139-5p were amplified by nested PCR and cloned into the region directly downstream of a CMV promoter-driven firefly luciferase cassette in the pcDNA3.0 vector (pLUC). The mutant $3^{\prime} U T R$ s of AMFR and NOTCH1, which carry the mutated sequence in the complementary site of the seed region of miR-139$5 p$, were generated from their $3^{\prime} U T R-W T$ plasmids by overlapextension PCR. The sequences of the wild-type and mutant 3'UTRs were confirmed by DNA sequencing. The open reading frame (ORF) of AMFR was amplified via nested PCR and cloned into the PWPXL vector. The intracellular domain of the NOTCH1 (ICN) plasmid pSINEF2-ICN was kindly provided by Prof. Lei Dong (State Key Laboratory of Pharmaceutical Biotechnology, School of Life Sciences, Nanjing University). The primers and endonuclease sites used for the vector constructs are shown in the Supplementary Data (Table S5).

\section{Oligonucleotide transfection}

MiR-139-5p mimics and inhibitors (anti-miR-139-5p, chemically modified antisense oligonucleotides designed to specifically target mature miR-139-5p), as well as their corresponding negative control (NC), were synthesised at Ribobio (Guangzhou, China). AMFR and NOTCH1 small interfering RNA (si-AMFR target sequence: 5'-GTACATACATCTCCTACAA-3'; siNOTCH1: 5'-GUCCAGGAAAC AACUGCAATT-3') were purchased from GenePharma (Shanghai, China). Oligonucleotide transfection was performed using Lipofectamine 2000 reagents (Invitrogen, USA) according to the manufacturer's instructions.

\section{Lentivirus production and transduction}

Lentivirus production and transduction was performed as described previously (Huang et al. 2011).

\section{Cell migration and invasion assay}

For the migration assays, $1 \times 10^{5}$ HCT-116 or $5 \times 10^{4}$ LoVo cells (stably expressed miR-139 or vector control) in $200 \mu \mathrm{L}$ serum-free medium were placed into the top chamber of each insert (BD Biosciences, $\mathrm{NJ}$ ), and $800 \mu \mathrm{L}$ medium supplemented with $10 \%$ fetal bovine serum into under chamber, After 24 (HCT-116) or 8 (LoVo) h of incubation at $37^{\circ} \mathrm{C}$, cells adhering to the lower membrane were stained with $0.1 \%$ crystal violet containing $20 \%$ methanol, imaged, and counted using an IX71 inverted microscope (Olympus, Japan). For the cell invasion assay, the polycarbonate membranes of the upper compartment of the chambers were pre-coated with a matrix gel.

\section{Cell proliferation assay}

Cell proliferation was quantified using the Cell Counting Kit-8 (CCK8; Dojindo Laboratories, Japan) according to the manufacturer's Manual as described previously (Huang et al. 2011).

\section{Tumor formation assay in a nude mouse model}

Athymic male BALB/c nude mice were feeding under specific pathogen-free conditions in the Experimental Animal Department of
Fudan University. The assay protocols were approved by the Shanghai Medical Experimental Animal Care Commission. For the in vivo tumor formation assays, $4 \times 10^{6}$ LoVo cells stably expressing miR-139 or the vector control were subcutaneously injected into either flank of the same athymic male BALB/c nude mouse at 5 weeks of age ( $n=6$ for each group). After transplantation, the growth of the tumors was assessed twice a week. The mice were sacrificed after a period of 4-6 weeks, and the weight of subcutaneous tumors were calculated. For the in vivo metastasis assays, $2 \times 10^{6}$ LoVo cells stably expressing miR-139 or the control vector were suspended in $100 \mu \mathrm{L}$ of DMEM and were injected into the caudal vein of each nude mouse ( $n=10$ for each group). The nude mice were maintained under specific pathogen-free conditions in the Experimental Animal Department of Fudan University. The mice were sacrificed after a period of 5 weeks.

\section{Microarray analysis}

Expression profiling was performed using an Agilent human whole genome oligo microarray chip $(4 \times 44 \mathrm{~K})$ (Agilent, USA). A total of $5 \times$ $10^{5}$ LoVo cells were seeded in $6-\mathrm{cm}^{2}$ tissue culture plates and transfected with the miR-139-5p mimic or NC as described above. After propagation for $48 \mathrm{~h}$, total RNA was extracted for the expression profiling analyses. The microarray profiling was performed as described in our previous work (Wang et al. 2014).

\section{Luciferase assay}

Approximately 5000 HEK-293T cells per well were plated into 96-well plates and were cotransfected with $50 \mathrm{nmol} / \mathrm{L}$ of $\mathrm{miR}-139-5 \mathrm{p}$ mimic (or NC), $50 \mathrm{ng}$ of the luciferase reporter, and $5 \mathrm{ng}$ of the $\mathrm{pRL}-$ CMV Renilla luciferase reporter using $0.5 \mu \mathrm{L}$ per well Lipofectamine 2000 (Invitrogen, USA). After $48 \mathrm{~h}$ incubation, the firefly and Renilla luciferase activities were quantified using a dual-luciferase reporter assay (Promega, USA).

\section{Western blot}

Cellular proteins were extracted and separated in SDS-PAGE gels, first separated by $10 \%$ sodium dodecyl sulphate-polyacrylamide gel electrophoresis and then transferred to nitrocellulose membranes (Bio-Rad Laboratories, USA). The membranes were blocked with $5 \%$ nonfat milk and incubated with a mouse anti-AMFR polyclonal antibody at a dilution of 1:500 (Abcam, USA), a rabbit anti-activated NOTCH1 antibody (Abcam, USA), or a mouse anti-beta-actin monoclonal antibody at a dilution of 1:1000 (Sigma, USA). The membranes were subsequently incubated with a goat anti-mouse horseradish peroxidase secondary antibody (Sigma, USA). The protein complex was detected using enhanced chemiluminescence reagents (Pierce, France).

\section{Immunohistochemical staining}

Tissue arrays were constructed using 134 paired CRC tissues and NCTs. Immunohistochemical staining was performed on $4 \mu \mathrm{m} \mathrm{sec-}$ tions of paraffin-embedded tissues to determine the expression level of AMFR protein. In brief, the slides were incubated in AMFR and NOTCH1 antibody diluted 1:200 at $4^{\circ} \mathrm{C}$ overnight. The subsequent 
steps were performed using the EnVision ${ }^{\mathrm{TM}}$ FLEX High $\mathrm{pH}$ visualisation system according to the manufacturer's instructions (DAKO, Demark). The scoring of AMFR and NOTCH1 was performed as previous described (Tsai et al. 2007; Skrtic et al. 2010).

\section{Statistical analyses}

The results are presented as the mean values \pm SEM. The data were subjected to Student's $t$-tests, the Mann-Whitney $U$ test or the Kruskall-Wallis test unless otherwise specified ( $X^{2}$ test, Spearson's correlation). The overall survival curves were plotted according to the Kaplan-Meier method, with the log-rank test applied for comparisons. A $P$ value of less than 0.05 was considered statistically significant. SPSS 16.0 package (IBM, USA) and Graphpad prism 5.0 software (GraphPad Software, USA) were used for statistical analyses and scientific graphing, respectively.

\section{ACKNOWLEDGEMENTS}

This work was supported by Grants from the National Natural Science Foundation of China (Grant Nos. 81071791, 81000867, and 81272299) and Medical Key Professionals Program of Jiangsu Province (RC2011031).

\section{ABBREVIATIONS}

AMFR, autocrine motility factor receptor; CRC, colorectal carcinoma; miRNA, microRNA; qRT-PCR, quantitative reverse transcription polymerase chain reaction; siRNA, small interfering RNA; $3^{\prime} U T R$, $3^{\prime}$ untranslated region.

\section{COMPLIANCE WITH ETHICS GUIDELINES}

Mingxu Song, Yuan Yin, Jiwei Zhang, Binbin Zhang, Zehua Bian, Chao Quan, Leyuan Zhou, Yaling Hu, Qifeng Wang, Shujuan Ni, Bojian Fei,, Weili Wang, Xiang Du, Dong Hua, and Zhaohui Huang declare that they have no conflict of interest.

All institutional and national guidelines for the care and use of laboratory animals were followed. All procedures followed were in accordance with the ethical standards of the responsible committee on human experimentation (institutional and national) and with the Helsinki Declaration of 1975, as revised in 2000 (5). Informed consent was obtained from all patients for being included in the study.

\section{OPEN ACCESS}

This article is distributed under the terms of the Creative Commons Attribution License which permits any use, distribution, and reproduction in any medium, provided the original author(s) and the source are credited.

\section{REFERENCES}

Asangani IA, Rasheed SA, Nikolova DA, Leupold JH, Colburn NH, Post S, Allgayer H (2008) MicroRNA-21 (miR-21) post-transcriptionally downregulates tumor suppressor $\mathrm{Pdcd} 4$ and stimulates invasion, intravasation and metastasis in colorectal cancer. Oncogene 27:2128-2136

Bao W, Fu HJ, Xie QS, Wang L, Zhang R, Guo ZY, Zhao J, Meng YL, Ren XL, Wang T et al (2011) HER2 interacts with CD44 to upregulate CXCR4 via epigenetic silencing of microRNA-139 in gastric cancer cells. Gastroenterology 141(2076-2087):e2076

Bartel DP (2004) MicroRNAs: genomics, biogenesis, mechanism, and function. Cell 116:281-297

Bouyssou JM, Manier S, Huynh D, Issa S, Roccaro AM, Ghobrial IM (2014) Regulation of microRNAs in cancer metastasis. Biochim Biophys Acta 1845(2):255-265

Bu P, Chen KY, Chen JH, Wang L, Walters J, Shin YJ, Goerger JP, Sun J, Witherspoon M, Rakhilin N et al (2013) A microRNA miR34a-regulated bimodal switch targets Notch in colon cancer stem cells. Cell Stem Cell 12:602-615

Chen X, Guo X, Zhang H, Xiang Y, Chen J, Yin Y, Cai X, Wang K, Wang G, Ba $Y$ et al (2009) Role of miR-143 targeting KRAS in colorectal tumorigenesis. Oncogene 28:1385-1392

Chiu CG, St-Pierre P, Nabi IR, Wiseman SM (2008) Autocrine motility factor receptor: a clinical review. Expert Rev Anticancer Ther 8:207-217

Chu D, Li Y, Wang W, Zhao Q, Li J, Lu Y, Li M, Dong G, Zhang H, Xie $\mathrm{H}$ et al (2010) High level of Notch1 protein is associated with poor overall survival in colorectal cancer. Ann Surg Oncol 17:13371342

Esquela-Kerscher A, Slack FJ (2006) Oncomirs-microRNAs with a role in cancer. Nat Rev Cancer 6:259-269

Fang S, Ferrone M, Yang C, Jensen JP, Tiwari S, Weissman AM (2001) The tumor autocrine motility factor receptor, gp78, is a ubiquitin protein ligase implicated in degradation from the endoplasmic reticulum. Proc Natl Acad Sci USA 98:1442214427

Gu W, Li X, Wang J (2013) miR-139 regulates the proliferation and invasion of hepatocellular carcinoma through the WNT/TCF-4 pathway. Oncol Rep 31(1):397-404

Guo J, Miao Y, Xiao B, Huan R, Jiang Z, Meng D, Wang Y (2009) Differential expression of microRNA species in human gastric cancer versus non-tumorous tissues. J Gastroenterol Hepatol 24:652-657

Guo H, Hu X, Ge S, Qian G, Zhang J (2012) Regulation of RAP1B by miR-139 suppresses human colorectal carcinoma cell proliferation. Int J Biochem Cell Biol 44:1465-1472

Hellstrom M, Phng LK, Hofmann JJ, Wallgard E, Coultas L, Lindblom P, Alva J, Nilsson AK, Karlsson L, Gaiano N et al (2007) DII4 signalling through Notch1 regulates formation of tip cells during angiogenesis. Nature 445:776-780

Hristova NR, Tagscherer KE, Fassl A, Kopitz J, Roth W (2013) Notch1-dependent regulation of p27 determines cell fate in colorectal cancer. Int J Oncol 43:1967-1975

Hu YY, Zheng MH, Zhang R, Liang YM, Han H (2012) Notch signaling pathway and cancer metastasis. Adv Exp Med Biol 727:186-198

Huang Z, Huang S, Wang Q, Liang L, Ni S, Wang L, Sheng W, He X, Du X (2011) MicroRNA-95 promotes cell proliferation and targets sorting Nexin 1 in human colorectal carcinoma. Cancer Res 71:2582-2589 
Hurst DR, Edmonds MD, Welch DR (2009) Metastamir: the field of metastasis-regulatory microRNA is spreading. Cancer Res 69:7495-7498

Hwang WL, Jiang JK, Yang SH, Huang TS, Lan HY, Teng HW, Yang CY, Tsai YP, Lin CH, Wang HW et al (2014) MicroRNA-146a directs the symmetric division of Snail-dominant colorectal cancer stem cells. Nat Cell Biol 16:268-280

Jemal A, Bray F, Center MM, Ferlay J, Ward E, Forman D (2011) Global cancer statistics. CA Cancer J Clin 61:69-90

Jiang WG, Raz A, Douglas-Jones A, Mansel RE (2006) Expression of autocrine motility factor (AMF) and its receptor, AMFR, in human breast cancer. J Histochem Cytochem 54:231-241

Kanbe K, Chigira M, Watanabe H (1994) Effects of protein kinase inhibitors on the cell motility stimulated by autocrine motility factor. Biochim Biophys Acta 1222:395-399

Kawanishi K, Doki Y, Shiozaki H, Yano M, Inoue M, Fukuchi N, Utsunomiya T, Watanabe H, Monden M (2000) Correlation between loss of E-cadherin expression and overexpression of autocrine motility factor receptor in association with progression of human gastric cancers. Am J Clin Pathol 113:266274

Kho DH, Nangia-Makker P, Balan V, Hogan V, Tait L, Wang Y, Raz A (2013) Autocrine motility factor promotes HER2 cleavage and signaling in breast cancer cells. Cancer Res 73:14111419

Krishnan K, Steptoe AL, Martin HC, Pattabiraman DR, Nones K, Waddell N, Mariasegaram M, Simpson PT, Lakhani SR, Vlassov A et al (2013) miR-139-5p is a regulator of metastatic pathways in breast cancer. RNA 19:1767-1780

Li Y, VandenBoom TG 2nd, Kong D, Wang Z, Ali S, Philip PA, Sarkar $\mathrm{FH}$ (2009) Up-regulation of miR-200 and let-7 by natural agents leads to the reversal of epithelial-to-mesenchymal transition in gemcitabine-resistant pancreatic cancer cells. Cancer Res 69:6704-6712

Li RY, Chen LC, Zhang HY, Du WZ, Feng Y, Wang HB, Wen JQ, Liu $X$, Li XF, Sun $Y$ et al (2013) MiR-139 inhibits Mcl-1 expression and potentiates TMZ-induced apoptosis in glioma. CNS Neurosci Ther 19:477-483

Liu R, Yang M, Meng Y, Liao J, Sheng J, Pu Y, Yin L, Kim SJ (2013) Tumor-suppressive function of miR-139-5p in esophageal squamous cell carcinoma. PLoS One 8:e77068

Livak KJ, Schmittgen TD (2001) Analysis of relative gene expression data using real-time quantitative PCR and the 2(-Delta Delta C (T)) Method. Methods 25:402-408

Manikandan J, Aarthi JJ, Kumar SD, Pushparaj PN (2008) Oncomirs: the potential role of non-coding microRNAs in understanding cancer. Bioinformation 2:330-334

Miele L, Golde T, Osborne B (2006) Notch signaling in cancer. Curr Mol Med 6:905-918

Mo YY, Tang H, Miele L (2013) Notch-associated microRNAs in cancer. Curr Drug Targets 14:1157-1166

Nakamori S, Watanabe H, Kameyama M, Imaoka S, Furukawa H, Ishikawa O, Sasaki Y, Kabuto T, Raz A (1994) Expression of autocrine motility factor receptor in colorectal cancer as a predictor for disease recurrence. Cancer 74:1855-1862

Nicoloso MS, Spizzo R, Shimizu M, Rossi S, Calin GA (2009) MicroRNAs-the micro steering wheel of tumour metastases. Nat Rev Cancer 9:293-302

Reedijk M, Odorcic S, Zhang H, Chetty R, Tennert C, Dickson BC, Lockwood G, Gallinger S, Egan SE (2008) Activation of Notch signaling in human colon adenocarcinoma. Int J Oncol 33:12231229

Shen K, Liang Q, Xu K, Cui D, Jiang L, Yin P, Lu Y, Li Q, Liu J (2012) MiR-139 inhibits invasion and metastasis of colorectal cancer by targeting the type I insulin-like growth factor receptor. Biochem Pharmacol 84:320-330

Skrtic A, Korac P, Kristo DR, Ajdukovic Stojisavljevic R, Ivankovic D, Dominis M (2010) Immunohistochemical analysis of NOTCH1 and JAGGED1 expression in multiple myeloma and monoclonal gammopathy of undetermined significance. Hum Pathol 41:1702-1710

Sureban SM, May R, Mondalek FG, Qu D, Ponnurangam S, Pantazis P, Anant S, Ramanujam RP, Houchen CW (2011) Nanoparticle-based delivery of siDCAMKL-1 increases microRNA-144 and inhibits colorectal cancer tumor growth via a Notch-1 dependent mechanism. J Nanobiotechnol 9:40

Taniguchi K, Yonemura Y, Nojima N, Hirono Y, Fushida S, Fujimura T, Miwa K, Endo Y, Yamamoto H, Watanabe H (1998) The relation between the growth patterns of gastric carcinoma and the expression of hepatocyte growth factor receptor (c-met), autocrine motility factor receptor, and urokinase-type plasminogen activator receptor. Cancer 82:2112-2122

Tsai YC, Mendoza A, Mariano JM, Zhou M, Kostova Z, Chen B, Veenstra T, Hewitt SM, Helman LJ, Khanna C et al (2007) The ubiquitin ligase gp78 promotes sarcoma metastasis by targeting KAl1 for degradation. Nat Med 13:1504-1509

Wang L, Hou G, Xue L, Li J, Wei P, Xu P (2010) Autocrine motility factor receptor signaling pathway promotes cell invasion via activation of ROCK-2 in esophageal squamous cell cancer cells. Cancer Invest 28:993-1003

Wang Q, Huang Z, Guo W, Ni S, Xiao X, Wang L, Huang D, Tan C, $\mathrm{Xu} Q$, Zha $\mathrm{R}$ et al (2014) MicroRNA-202-3p Inhibits Cell Proliferation by Targeting ADP-Ribosylation Factor-like $5 \mathrm{~A}$ in Human Colorectal Carcinoma. Clin Cancer Res 20:1146-1157

Wong CC, Wong CM, Tung EK, Au SL, Lee JM, Poon RT, Man K, Ng IO (2011) The microRNA miR-139 suppresses metastasis and progression of hepatocellular carcinoma by down-regulating Rhokinase 2. Gastroenterology 140:322-331

Yanagawa T, Funasaka T, Tsutsumi S, Watanabe H, Raz A (2004) Novel roles of the autocrine motility factor/phosphoglucose isomerase in tumor malignancy. Endocr Relat Cancer 11:749759

Zhang L, Dong Y, Zhu N, Tsoi H, Zhao Z, Wu CW, Wang K, Zheng S, $\mathrm{Ng}$ SS, Chan FK et al (2014) microRNA-139-5p exerts tumor suppressor function by targeting NOTCH1 in colorectal cancer. Mol Cancer 13:124 